\title{
Optical Saturation of Strong Selective Reflection
}

\author{
Yakov A. Fofanov \\ Institute for Analytical Instrumentation RAS, St.-Petersburg, 190103, Russia \\ *Corresponding Author: yakinvest@yandex.ru
}

Copyright $@ 2013$ Horizon Research Publishing All rights reserved.

\begin{abstract}
Nonlinear selective reflection of obliquely incident laser beam (inclined geometry) has been investigated. The new structures were detected on the tops of strong reflection resonances under conditions of optical saturation of the D2-lines in the natural mixture of rubidium isotopes. The detected nonlinear structures have not saturation properties in usual sense and exist only in the close area of saturating power density values. This phenomenon can be useful for the amplification the nonlinear characteristics of the optical interface in order to reduce the fluctuations of selective reflected light.
\end{abstract}

Keywords Laser, Coherent Light, Selective Reflection, Nonlinear Optics, Light Noise, Spectroscopy

\section{Introduction}

The selective reflection (SR) i.e. the reflection from the dielectric - resonant atomic gas interface is known for a long time [1-3]. The interest in this phenomenon was related mainly to its applications for the spectroscopy, especially the spectroscopy of dense gases [2, 4-7]. Strong impulse for the study and application of the SR was done by the finding of sub-Doppler resonances [3, 8-15]. Selective reflection is also a good method for studies of the interaction of excited atoms with a solid surface including van der Waals interaction [16-18]. One of the useful applications of SR is the stabilization of the laser frequency [19-22].

There are two basic schemes that are used to study the SR: traditional single beam scheme (SB-scheme) [1-18], and pump-probe scheme (PP-scheme) that use pump and probe beams with independent frequencies [23-28]. Selective reflection is investigated in a wide range from normal up to large light incidence angles [29,30]. Additional advantages are provided under investigation on conditions of total internal reflection (TIR) because the interaction atoms with evanescent wave takes place at the vicinity of dielectric surface [30-34]. The study of nonlinear SR reveals some important new details of the resonant interaction of atoms and fields near the interface [13,23,29,35-38]. The novel features and prospects of development of the high sensitive laser spectroscopy has recently made possible with intensive investigations of SR as compare with transmission in "nanometric" gas cells (super-thin cells) [39-41].

Separately, it is noted that the use of semiconductor lasers (diode lasers) is proved quite effective for the study of SR $[11,35]$. In PP-scheme the lasers diode are often used in combination with the lasers of other types [25,27]. The relatively high level of frequency fluctuations (phase noise) of semiconductor lasers was helpful for the development of diode lasers noise spectroscopy [35,42]. More detailed fluctuations (including quantum ones) of lasers diode as well as their use for the spectroscopy and precision optical measurements, including investigations of SR, are considered in [43-47].

Our study have confirmed a significant increase in the amplitude of SR resonances for large incidence angles, that had been observed previously in [30]. A contrast of the strongest refection resonances in SB-scheme exceeds 500\% at the moderate heating of reflecting cell [48]. In the present paper is described in more detail than in [49], the novel nonlinear phenomenon under strong SR from the interface glass - rubidium vapour in an inclined geometry.

\section{Experimental Methods}

The most significant elements of the experimental setup (SB-scheme) are shown in Figure 1. Radiation of continuous wave semiconductor laser is directed on the reflection and absorbing cells by means of beam splitter BS. In reflection cell laser radiation interacts with a glass - rubidium vapor interface. The plane of polarization of the laser light is parallel to the plane of incidence. Reflection and absorption cells are filled with natural abundance of ${ }^{85} \mathrm{Rb}$ and ${ }^{87} \mathrm{Rb}$ isotopes.

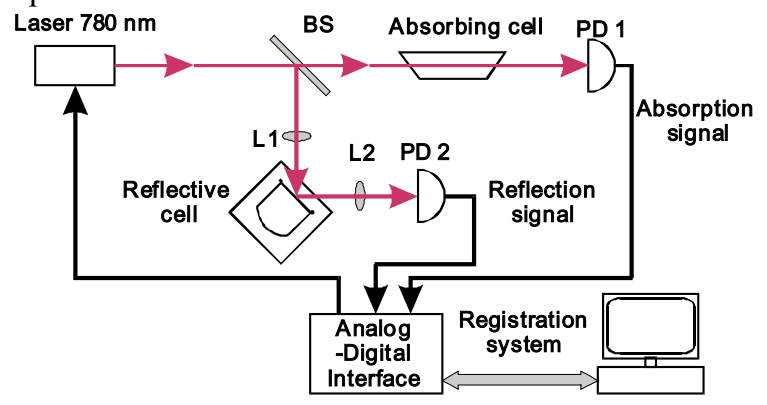

Figure 1. Experimental Setup 
Lenses L1, L2 are designed for focusing the laser radiation on the surface to be studied, and for matching parameters of the reflected beam with an aperture of the photodetector. Laser radiation is detected by photodiodes PD1, PD2, and than the absorption and reflection signals are transmitted in the registration system. Absorption signal is used for the calibration of the laser frequency.

Figure 2 gives the qualitative picture selective reflection of coherent light in inclined geometry. The angle of incidence is determined only by the angular position of a reflecting cell. Light is polarized in the incidence plane. At the presence of a resonance gas, the critical angle of TIR and Brewster angle depend on the detuning of the optical frequency relative to the center of line. This leads to significant changes in the intensity of reflected light when adjusting the frequency. In particular a resonant approaching of the critical angle of TIR to the angle of incidence is accompanied by a sharp increase in the reflection coefficient.

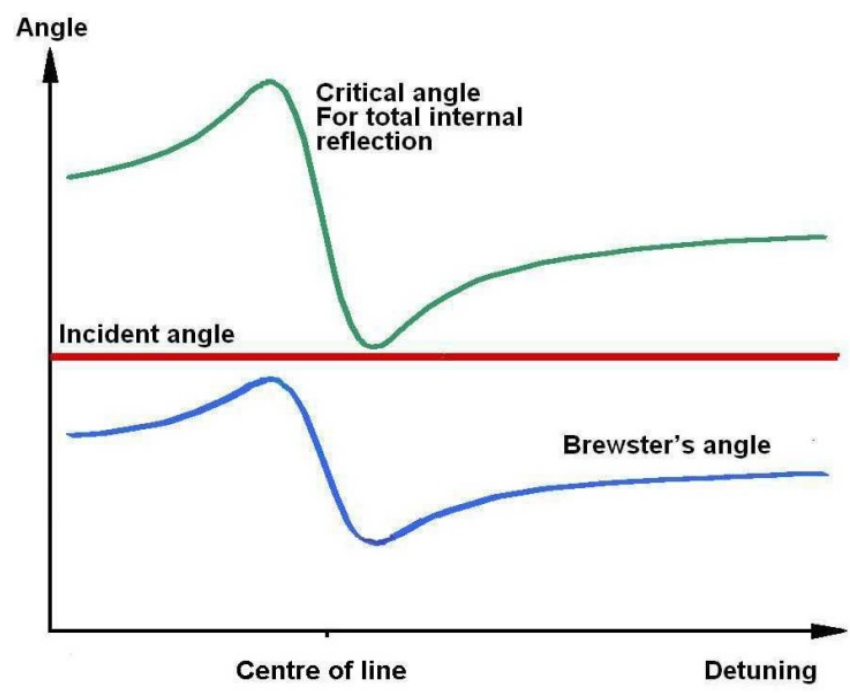

Figure 2. Reflection conditions

\section{Investigations Results}

\subsection{Strong Nonlinear Selective Reflection}

Curves $\mathrm{W}_{1}-\mathrm{W}_{3}$ in Figure 3 show typical experimental dependences of the reflection coefficient on the laser frequency in the inclined geometry. Insert in Figure 3 shows energy levels and transitions for rubidium isotopes at $780 \mathrm{~nm}$. One can see two transitions for each isotope. In our experiments others transitions are hidden Doppler broadening.

Spectra $\mathrm{W}_{1}-\mathrm{W}_{3}$ are obtained at incidence angle of less than off-resonant critical angle for TIR. Curve $\mathrm{W}_{1}$ corresponds to the surface power density at the interface that far from saturating value. It is clearly visible the peaks of the SR (reflective resonances) a', A', B', and b'. On the tops of resonances $\mathrm{A}^{\prime}, \mathrm{B}^{\prime}, \mathrm{b}^{\prime}$ the reflection conditions are close to the TIR. The main features of the strong unsaturated SR in inclined geometry in more detail are given in [48].

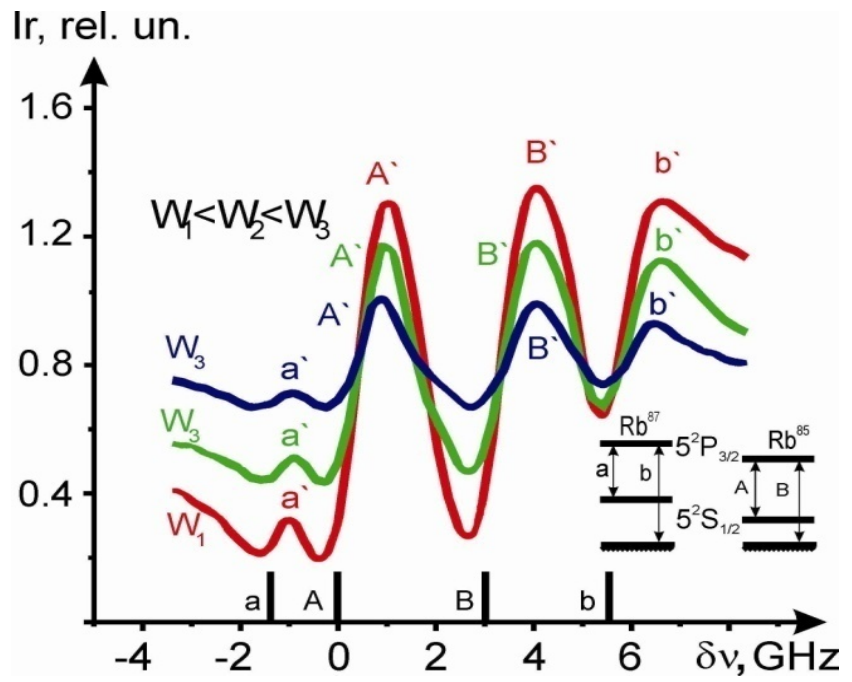

Figure 3. Strong nonlinear selective reflection

Curves $\mathrm{W}_{2}$ and $\mathrm{W}_{3}$ were obtained with an increase in surface power density of up to saturating values. Due to optical saturation of resonant transitions the reflection curves become smoother. Particularly interesting is the decrease in the coefficient of SR on the tops of the resonances A', B', and $\mathrm{b}^{\prime}$. This phenomenon has been used to reduce a fluctuations of the reflected light $[50,51]$. In the following section will be continued a discussion of the nonlinear properties of strong SR.

\subsection{The New Nonlinear Structures}

In the process of our experiments it is found that by optical saturation of SR in inclined geometry on the reflection spectra appear previously unknown structures. More detailed studies have shown also that the observed structures have the appearance of characteristic peaks on the tops of the reflection resonances. Depending on experimental conditions, are observed a series of peaks, as well as the single peaks.

One of the typical reflection spectra with series of peaks is presented in Figure 4. Peaks $\mathrm{P}_{1}, \mathrm{P}_{2}$, and $\mathrm{P}_{3}$ are good seen at the top of the resonance $\mathrm{A}^{\prime}$. Contrast of peaks $\mathrm{P}_{1}$ and $\mathrm{P}_{2}$ is about $1,5 \%$, the distance between peaks is about $150 \mathrm{MHz}$. At the top of the resonance B ' begins to form a weaker peak $\mathrm{P}_{4}$. Sufficiently good reproducibility of the spectra in Figure 4 can be seen from a comparison between two scans from a series of 20 ones, taken in the same experimental conditions.

In Figures 5 and 6 are shown an individual peaks located on the components $\mathrm{A}^{\prime}$ and $\mathrm{B}^{\prime}$ respectively. More detailed image of the single peak can be seen in Figure 7. Contrast of this peak is about $6 \%$. Its width is approximately $0.16 \mathrm{GHz}$.

The main difference between the experimental conditions under which are observed a series of peaks or an individual peaks, is in a different position of the lens L1 (see Figure 1). The transition from the spectrum shown in Figure 4 to the spectra in Figure 5, 6 is achieved by the move the lens by 
approximately 1-3 mm closer to the reflecting cell.

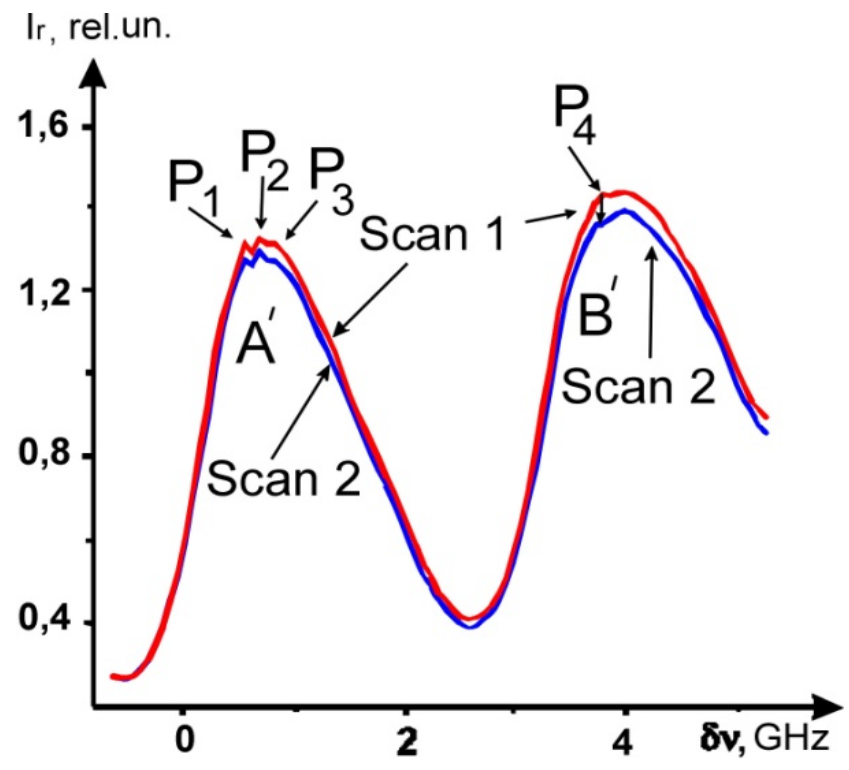

Figure 4. Series of peaks

A comparable movement is necessary for the transformation the spectrum in Figure 5 to the spectrum in Figure 6. The main parameter that changes with these moves is the surface power density $\mathrm{W}$ at the resonant interface. According to our estimates these changes of $\mathrm{W}$ are about $30 \%$.

It is quite possible also that the specified movement of the lens can cause the displacement of the light spot on the reflection cell window. As well not excluded small changes of the incidence angle and the turns of the plane of the incident light polarization. In this connection it should be noted that near TIR even small changes in the geometry of the experiment entail appreciable changes of the reflection conditions [48].

\section{Conclusion}

In this section, we compare the obtained data with the known results and try to give the possible explanation of the nature of observed nonlinear phenomenon. One of the assumptions can be that the peaks in Figures $4-7$ are well known sub-Doppler resonances in SR [8-11]. However, it can not be so at least in several reasons. First, sub-Doppler resonances exist in zero detunings relative to the center of line, and only at angles of incidence close to the zero. Further, with an increasing of power density, these resonances begin to saturate [13,29,35-37].

Nonlinear structures described here, have not usual saturation properties. They are formed only in a fairly narrow range of values of the surface power density. Beside this, they appear at high angles and near the maxima of SR, which are strongly displaced with respect to the centers of spectral lines. For example, the maximum resonance $A^{\prime}$ of the reflection spectra in Figure 5 is shifted on about $0.8 \mathrm{GHz}$ in a high-frequency side from the center of the component A. Thus, the structures observed here are in no way can be associated with sub-Doppler resonances in SR, described in [8-15].

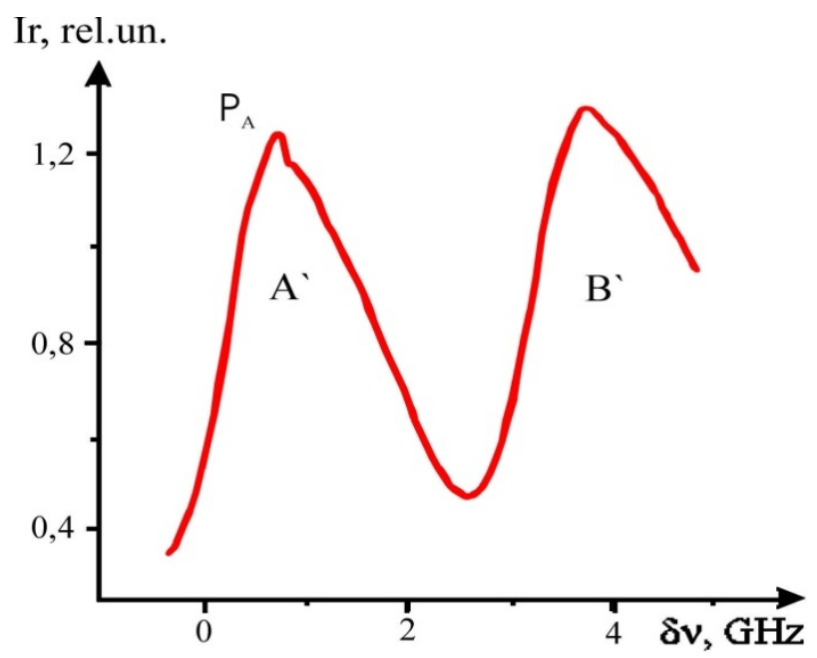

Figure 5. Single peak on the resonance A

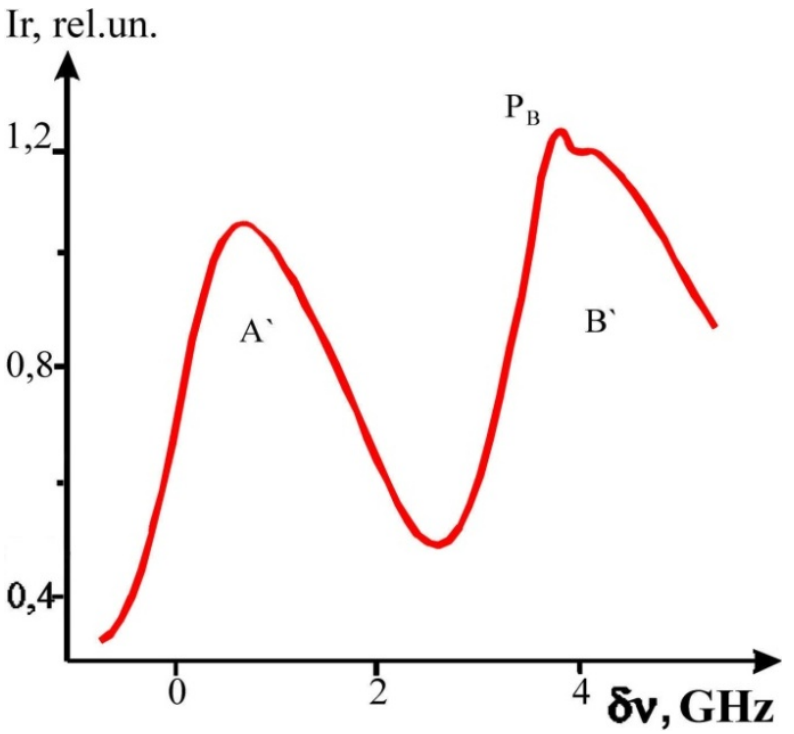

Figure 6. Single peak on the resonance B

It seems appropriate to try to compare the observed here resonances with narrow Doppler-free resonances in SR under conditions of TIR [32]. And here we also are finding the same difference: sub-Doppler resonances that reported in [32], exist only at the centers of the corresponding optical transitions.

Finally it should be noted external similarity of some features of the SR describe here and reflectance spectra of sub-micron thick vapor cell $[39,40]$. From the comparison of Figure 5 in [40] and Figure 7 in the present paper, we can see that in both cases the shapes of the peaks are very alike to each other. At the same time, here is the same important distinction - in the spectra of the SR from ultrathin cells the sub-Doppler resonances are observed at the frequencies of optical transitions. 


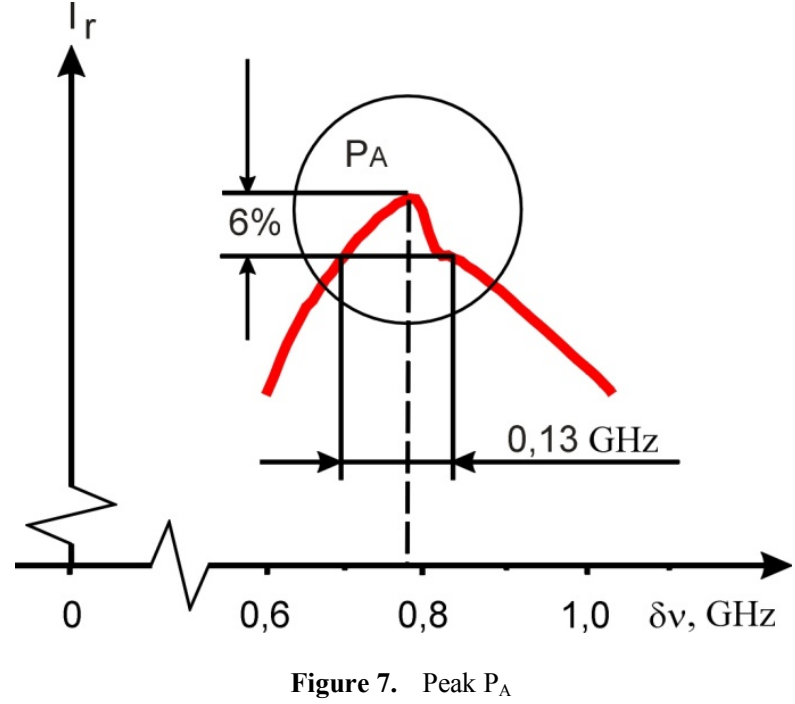

One of the possible explanations for the observed structures take into account the existence of absorption in the resonance gas. This absorption leads to a gradual decrease in the intensity of transmitted light as it propagates from the interface. The result is a nonuniform optical saturation and therefore a spatial inhomogeneity of the gas refractive index near the window of the cell. As a consequence, the investigated interface can exhibit the optical properties similar in some respects to properties of ultrathin cells.

Some chance of success has also an another assumption. It lies in the fact that the observed structures are associated with the manifestation in the system under investigation the properties of optical bistability [52]. The saturation of reflection leads to an increase the intensity of transmitted light. Because the atoms are found in transmitted light, the increase of its intensity leads to further optical saturation of resonant gas and accordingly to the additional decrease of the reflection. Abrupt decline in the intensity on the right side of the peak in Figure 7 may be explained by the realization of this process.

Naturally, the above considerations can not be exhaustive. There can well be other assumptions (models) that in more detail consider all aspects of the resonant interaction of atoms with coherent radiation near the surface $[18,37,53,54]$.

\section{Acknowledgements}

Author is very grateful to V. A. Sautenkov, V. L. Velichanskii, V. V. Vasiliev, N. N. Yakobson, and V. V. Khromov for their highly useful consultations during preparing the experiment.

\section{REFERENCES}

[1] R. W. Wood. The selective reflection of monochromatic light by mercury vapor, Philos. Mag., Vol.18, No.187, 1909.
[2] A.C. Lauriston, H.L. Welsh. Selective reflection from the vapors of the alkali metals, Canad. J. Phys., Vol.39, 217-226, 1951.

[3] J. L. Cojan. Contribution a l'etude de la reflection selective sur les vapeurs de mercure de la radiation de resonance du mercure, Ann. Phys. (Paris), Vol.9, 385-440, 1954.

[4] C. L. Chen, A. V. Phelps. Self-broadening of cesium resonance lines at 8521 and $8944 \AA$, Physical Review, Vol.173, No.1, 62-69, 1968.

[5] B. Senitzky. Optical filter using a vapor mirror, Appl. Phys. Lett., Vol.24. No.2, 68-70, 1974.

[6] J. Guo, A. Gallagher, J. Cooper. Lorentz-Lorenz shift in an inhomogeneously broadened medium, Opt. Commun., Vol.131, No.4-6, 219-222, 1996.

[7] P. Wang, A. Gallagher, J. Cooper. Selective reflection by Rb, Phys. Rev., Vol.A 56, 1598-1606, 1997.

[8] J. P. Woerdman M. F. H. Schuurmans, Spectral narrowing of selective reflection from sodium vapour, Opt. Commun., Vol. 14, 248-251, 1975.

[9] M. F. H. Schurmans. Spectral narrowing of selective reflection, J. Physique, Vol.37, 469-485, 1976.

[10] A. L. J. Burgmans, J. P. Woerdman. Selective reflection from sodium vapour at low densities, J. Physique, Vol.37, 677-681, 1976.

[11] V. A. Sautenkov, V. L. Velichasnskii, A. S. Zibrov, V. I. Luk'yanov, V. V. Nikitin, D. A. Tyurikov. Intra-Doppler resonances of the cesium D2 line in a selective specular reflection profile, Sov. J. Quantum Electron. Vol.11, No.9, 1131-1134, 1981.

[12] A. M. Akul'shin, V. L. Velichanskii, A. S. Zibrov, V. V. Nikitin, V. V. Sautenkov, E. K. Yurkin, N. V. Senkov. Collisional broadening of intra-Doppler resonances of selective reflection on the D2 line of cesium, Sov. Phys.-JETP Letters, Vol.36, No.7, 303-307, 1982.

[13] T. A. Vartanyan. Resonant reflection of intense optical radiation from a low-density gaseous medium, Sov. Phys.-JETP, Vol.61, No.4, 674-677, 1985.

[14] Xiao Lian-tuan, Zhao Jian-ming, Li Chang-yong, Jia Suo-tang, and Zhou Guo-sheng. Precise measurement of density-dependent shift by wavelength modulation reflection Spectroscopy, Chinese Physics, Vol.10, No.8, 716-719, 2001.

[15] V. A. Sautenkov, T. S. Varzhapetyan, H. Li, D. Sarkisyan, M. O. Scully. Selective reflection of a laser beam from a dilute rubidium vapor, Journal of Russian Laser Research (Moscow), Vol.31, No.3, 270-275, 2010.

[16] M. Chevrollier, J. G. de Souza, D. Bloch, M. Fichet, M. Ducloy. Selective reflection spectroscopy of a resonant vapor at the interface with a metallic layer, Phys. Rev., Vol.E63, $046610,2001$.

[17] H. Failache, S. Saltiel, M. Fichet, D. Bloch, M. Ducloy. Resonant coupling in the van der Waals interaction between an excited alkali atom and a dielectric surface: an experimental study via stepwise selective reflection spectroscopy, Eur. Phys. J., Vol.D23, 237-255, 2003.

[18] D. Bloch, M. Ducloy. Atom-wall interaction, Advances in At., 
Mol., and Optical Phys., Vol.50, No.1, 2005.

[19] V. S. Letokhov. Selective feedback for broadband lasers, Kr. Soobshch. Fiz. FIAN (Moscow), Vol.11, 14-17, 1970.

[20] B. Bolger, C. Weysenfeld. Locking of organic dye laser frequency to atomic resonance lines, IEEE Journal of Quantum Electronics, Vol.8, No.6, 529 529, 1972.

[21] V. L. Velichansky, A. S. Zibrov, V. V. Nikitin, V. A. Sautenkov, V. K. Malyshev, G. G. Kharisov. Semiconductor laser with $133 \mathrm{Cs}$ vapor external selective mirror, Sov. J. Quantum Electron., Vol.8, No.7, 836-840, 1978.

[22] T. Ito, T. Hashi, T. Yabuzaki. Frequency stabilization of an AlGaAs laser using selective reflection spectrum, Opt. Commun., Vol.82, No.5-6, 473-476, 1991.

[23] O. A. Rabi, A. Amy-Klein, S. Saltiel, M. Ducloy. Three-Level Non-linear Selective Reflection at a Dielectric/Cs Vapour Interface, Europhys. Lett., Vol.25, No.8, 579,1994

[24] H. Van Kampen, V. A. Sautenkov, E. R. Eliel, J. P. Woerdman. Probing the spatial dispersion in a dense atomic vapor near a dielectric interface, Phys. Rev. Vol.A58, No.6, 4473-4478, 1998.

[25] Yan-Ting Zhao, Jie Ma, Li-Rong Wang, Jian-Ming Zhao, Lian-Tuan Xiao, Suo-Tang Jia. Spectral hole-burning in pump-probe studies of nonlinear selective reflection spectroscopy, J. Phys. B: At. Mol. Opt. Phys., Vol.38, 3037-3042, 2005. doi: 10.1088/0953-4075/38/16/014

[26] Zhao Yan-Ting, Zhao Jian-Ming, Huang Tao, Xiao Lian-Tuan and Jia Suo-Tang. Experimental Observation of Autler-Townes Splitting in Sub-Doppler Selective Reflection Spectroscopy, Chinese Phys. Lett. Vol.22, 1668, 2005.

[27] V. A. Sautenkov, Y. V. Rostovtsev, E. R. Eliel. Observation of narrow Autler-Townes components in the resonant response of a dense atomic gas, Phys. Rev., Vol.A 78, No.1, $013802,2008$.

[28] H. Li, V. A. Sautenkov, Y. V. Rostovtsev, M. O. Scully. Excitation dependence of resonance line self-broadening at different atomic densities, J. Phys. B: At. Mol. Opt. Phys., Vol.42, 065203, 2009. doi: 10.1088/0953-4075/42/6/065203

[29] G. Nienhuis, F. Schuller, M. Ducloy. Nonlinear selective reflection from an atomic vapor at arbitrary incidence angle, Phys. Rev., Vol.A 38, 5197-5205, 1988.

[30] A. M. Akul'shin, V. L. Velichanskii, A. I. Zherdev, A. S. Zibrov, V. U. Malakhova, V. V. Nikitin, V. A. Sautenkov, G. G. Kharisov. Selective reflection from the glass-gas interface at high angles, Sov. J. Quantum Electron., Vol.19, No.3, 416-419, 1989.

[31] P. Boissel, F. Kerherve. Absorption de lumiere par des atomes dans une onde evanescente, Opt. Commun., Vol.37, No.6, 397-402, 1981.

[32] P. Simoneau, B. S. Le, C. B. De Arauio, D. Bloch, I. R. Lios Leite, M. Ducloy. Doppler-free evanescent wave spectroscopy, Opt. Commun., Vol.59, No.2, 103-106, 1986.

[33] V. G. Bordo, H.-G. Rubahn. Two-photon evanescent-wave spectroscopy of alkali-metal atoms, Phys. Rev., Vol.A 60, 1538-1548, 1999.

[34] R. Kondo, S. Tojo, T. Fujimoto, M. Hasuo. Shift and broadening in attenuated total reflection spectra of the hyperfine-structure-resolved D2 line of dense rubidium vapor, Phys. Rev., Vol.A73, 062504, 2006.

[35] Yu. A. Bykovskii, V. L. Velichanskii, V. K. Egorov, A. S. Zibrov, V. A. Maslov. Optical pumping and nonlinear effects in the spectroscopy of the cesium D2 line, JETP Letters, Vol.19, No.11, 345-346, 1974.

[36] A. M. Akul'shin, T. A. Vartanyan, V. L. Velichanskii, R. G. Gamidov, V. V. Sautenkov. Nonlinear effects in selective reflection from resonant gas, Izv. Akad. Nauk SSSR, Ser. Fiz. (Moscow), Vol.53, No.6, 1122-1124, 1989.

[37] J. Guo, J. Cooper, A. Gallagher, M. Lewenstein. Theory of selective reflection spectroscopy. Opt. Commun., Vol.110, 197-208, 1994.

[38] R. Walser, J. Cooper, P. Zoller. Saturated absorption spectroscopy using diode-laser phase noise, Phys. Rev., Vol.A 50, 4303-4309, 1994.

[39] D. Sarkisyan, D. Bloch, A. Papoyan, M. Ducloy. Sub-Doppler spectroscopy of sub-micron thin Cs vapour layer, Opt. Comm., Vol.200, 201-208, 2001.

[40] G. Dutier, A. Yarovittskii, S. Saltiel, A. Papoyan, D. Sarkisyan, D. Bloch, M. Ducloy. Collapse and revival of a Dicke-type coherent narrowing in a sub-micron thick vapor cell transmission spectroscopy, Europhys. Lett., Vol.63, No.1, 35-41, 2003.

[41] I. Hamdi, P. Todorov, A. Yarovitski, G. Dutier, I. Maurin, S. Saltie, Y. Li, A. Lezama, T. Varzhapetyan, D. Sarkisyan, M.-P. Gorza, M. Fichet, D. Bloch, M. Ducloy. Laser Spectroscopy with Nanometric Gas Cells: Distance Dependence of Atom-Surface Interaction and Collisions under Confinement, Laser Physics, Vol.15, No.7, 987-996, 2005.

[42] D. H. McIntyre, C. E. Fairchild, J. Cooper, R. Walser. Diode-laser noise spectroscopy of rubidium, Optics Letters, Vol.18, No.21, 1816-1818, 1993.

[43] K.Peterman. Laser Diodes Modulation and Noise, Kluwer Academic Publshers, 1991.

[44] C. E. Wieman, L. Hollberg. Using diode lasers for atomic physics, Rev. Sci. Instr., Vol.62., 1-20, 1991.

[45] I. V. Sokolov, Ya. A. Fofanov. On the Admissible Natural Linwidth of Laser Radiation Necessry for the Observation of Squeezing in the Case of Self-Phase Modulation in a Resonant Medium, Optics and Spectroscopy, Vol.81, No.4, 635-637, 1996.

[46] A. S. Trifonov. When May a Diode Laser be Regarded as a Single-Mode Laser from the Viewpoint of Quantum Optics? Optics and Spectroscopy. Vol. 86, No.1, 121-125, 1999.

[47] Ya. A. Fofanov, I. V. Sokolov. Sub-Poissonian single-mode lasing in a semiconductor laser with an external cavity, Journal of Optical Technology, Vol.70, No.1, 38-41, 2003.

[48] Ya. A. Fofanov. Selective reflection of obliquely incident polarized light, Sov. J. Quantum Electron., Vol.39, No.6, 585-590, 2009.

[49] Ya. A. Fofanov. New nonlinear phenomena under conditions of strong selective reflection. In ICONO 2010: International Conference on Coherent and Nonlinear Optics. Edited by Claude Fabre, Victor Zadkov, Konstantin Drabovich. 
Proceedings of SPIE. Vol. 7993. (SPIE, Bellingham, WA 2011) 799300 (2011); doi:10.1117/12.882693.

[50] Ya. A. Fofanov. Transformation of intensity fluctuations in nonlinear reflection of light. Optics and Spectroscopy, Vol.94, No.5, 802-804, 2003.

[51] Ya. A. Fofanov. Controlling the Intensity and Fluctuations of Light upon Its Selective Reflection, Optics and Spectroscopy, Vol.99, No.3, 457-458, 2005.
[52] H. M. Gibbs. Optical Bistability. Controlling Light With Light, Academic Press, Orlando, 1985.

[53] T. A. Vartanyan. Optical Control of Longitudinal Periodic Structures Near the Boundary of a Rarefied Resonance Medium, Optics and Spectroscopy, Vol.88, No.4, 564-567, 2000.

[54] V. A. Sautenkov. Line shapes of atomic transitions in excited dense gas, Laser Phys. Lett., Vol.8, No.11, 771-781, 2011. DOI 10.1002/lapl.201110070 771. 